\section{Checking in cell death}

The cell-cycle regulator p53 is mutated in half of all cancers and is indirectly inactivated in a large proportion of others. This means that pathways involved in p53-independent cell death represent important targets for the treatment of cancer. One such promising pathway is $\mathrm{p} 73$ mediated apoptosis in response to DNA damage, and Carol Prives and colleagues have identified some of the crucial components that activate p73.

To begin, the researchers established that TP73 mRNA accumulates in human cell tumour lines in response to DNA damage induced by various agents, including the chemotherapeutic drugs etoposide, camptothecin, daunorubicin and doxorubicin. They then turned to the two checkpoint kinases, $\mathrm{CHK} 1$ and CHK2, components of the DNAdamage checkpoint that are essential for the induction of cell-cycle arrest, DNA repair and apoptosis. Using small interfering RNAs to modulate the production of CHK1 and CHK2, they found that these proteins are required for the accumulation of both TP73 mRNA and $\mathrm{p} 73$ protein in response to DNA damage.

So how do CHK1 and CHK2 influence the transcription of TP73? The researchers were curious about the role of the transcription factor E2F1 because it is able to induce TP73 mRNA, it binds to the TP73 promoter in response to DNA damage,

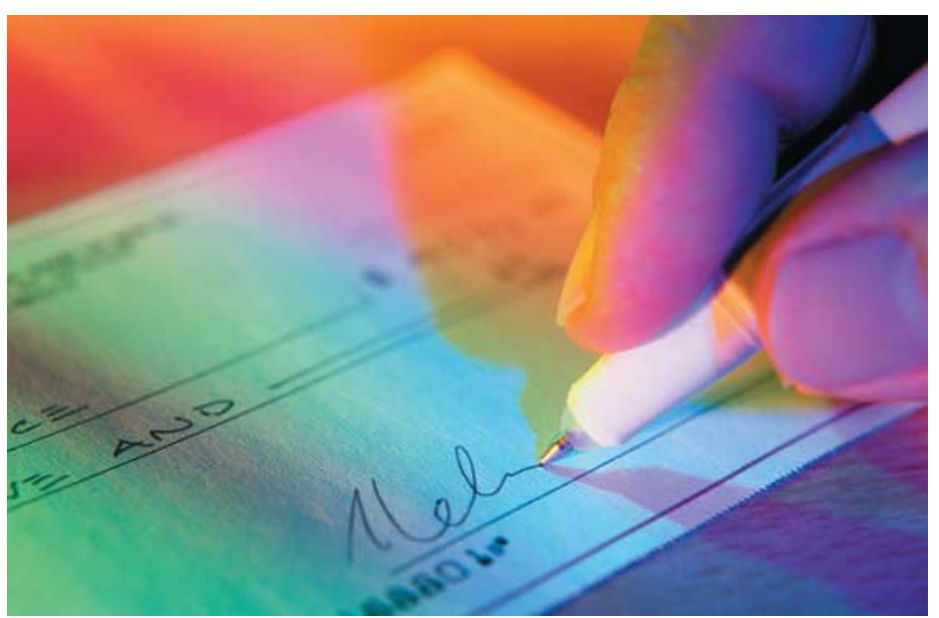

and because $\mathrm{p} 73$ is required for E2F1induced apoptosis. They discovered that E2F1 is stabilized by $\mathrm{CHK} 1$ and CHK2 in response to DNA damage, and, using an inducible mutant form of $\mathrm{E} 2 \mathrm{~F} 1$, showed that it is this $\mathrm{E} 2 \mathrm{~F} 1$ stabilization that results in the accumulation of p73. This establishes E2F1 as the crucial checkpointkinase-regulated transcription factor responsible for the activation of the TP73 promoter after DNA damage.

Finally, to verify that these four proteins act in a common pathway during p53-independent apoptosis, Prives and co-workers confirmed that the downregulation of any one of the four proteins - $\mathrm{CHK} 1, \mathrm{CHK} 2, \mathrm{E} 2 \mathrm{~F} 1$ or p73 - impedes the apoptosis that normally occurs in response to DNA damage. Having established the connection between DNA damage and TP73 transcription and stabilization, the authors raise the next pressing question: how does p73 go on to mediate p53-independent apoptosis? The authors propose new experiments to investigate these further potential targets for the treatment of cancer and speculate about the role of the p53-related protein - p63 — during p53-independent apoptosis. Jenny Bangham

6) References and links ORIGINAL RESEARCH PAPER Urist, M. Tanaka, T., Poyurovsky, M. V. \& Prives, P. p73 induction after DNA damage is regulated by checkpoint kinases, Chk1 and Chk2. Genes Dev. 15 Dec 2004 (doi:10.1101/gad.1221004)

\section{IN BRIEF}

\section{GENETICS}

\section{Fbxw7/Cdc4 is a p53-dependent, haploinsufficient} tumour suppressor gene.

Mao, J.-H. et al. Nature 432, 775-779 (2004)

Fbwx7 encodes a ubiquitin ligase associated with maintenance of chromosome stability. Mao et al. show that this gene is regulated by $\mathrm{p} 53$, and that its downregulation in mice leads to radiationinduced tumorigenesis. The authors propose that loss of FBWX7 activity leads to genetic instability through activation of the spindle-checkpoint protein Aurora-A.

\section{COLON CANCER}

15-Hydroxyprostaglandin dehydrogenase, a COX-2 oncogene antagonist, is a TGF- $\beta$ induced suppressor of human gastrointestinal cancers.

Yan, M. et al. Proc. Natl Acad. Sci. USA 14 Dec 2004 (doi:10.1073/pnas.0406142101)

Upregulation of cyclooxygenase-2 (COX2) is an early event in human colon tumorigenesis. Yan et al. report that colon cancer further targets the prostaglandin biogenesis pathway by downregulating expression of 15-hydroxyprostaglandin dehydrogenase (15-PGDH), an enzyme that antagonizes COX2. Re-expression of 15-PGDH in colon cancer cells inhibits their ability to form tumours in mice, and the authors show its expression in colon cells is induced by activation of the transforming growth factor- $\beta$ tumour-suppressor pathway.

\section{TUMOUR SUPPRESSORS}

Small molecule RITA binds to p53, blocks p53-HDM-2 interaction and activates p53 function in tumors.

Issaeva, N. et al. Nature Med. 10, 1321-1328 (2004)

In some cancers where $\mathrm{p} 53$ is wild type, its function is effectively inhibited due to degradation through the proteasome, often a result of the aberrant increased expression of its regulator, the ubiquitin ligase HDM2. Issaeva et al. have identified a small molecule, RITA, which prevents the interaction between HDM2 and $\mathrm{p} 53$, and induces massive apoptosis due to the stabilization of p53. RITA has shown significant antitumour effect in vivo and shows promise as a drug to target tumours with wild-type p53.

\section{CELL ADHESION}

Specific deletion of focal adhesion kinase suppresses tumour formation and blocks malignant progression.

McLean, G. W. et al. Genes Dev. 15 Dec 2004 (doi:10.1101/gad.316304)

Loss of cellular focal adhesions is crucial to the development and progression of invasive cancer. One component of focal adhesions — focal-adhesion kinase (FAK) — has been associated with tumour progression but has never definitively been shown to be a regulatory factor. Using a regulatable FAK allele, McLean et al. show that FAK prevents apoptosis and its loss suppresses skin tumour formation and progression in vivo. 